\title{
DECIGO and DECIGO pathfinder
}

This article has been downloaded from IOPscience. Please scroll down to see the full text article.

2010 Class. Quantum Grav. 27084010

(http://iopscience.iop.org/0264-9381/27/8/084010)

View the table of contents for this issue, or go to the journal homepage for more

Download details:

IP Address: 194.94.224.254

The article was downloaded on 10/09/2012 at 10:23

Please note that terms and conditions apply. 


\title{
DECIGO and DECIGO pathfinder
}

\author{
Masaki Ando ${ }^{1}$, Seiji Kawamura ${ }^{2}$, Naoki Seto ${ }^{1}$, Shuichi Sato ${ }^{3}$, \\ Takashi Nakamura $^{1}$, Kimio Tsubono $^{4}$, Takeshi Takashima ${ }^{5}$, \\ Ikkoh Funaki $^{5}$, Kenji Numata ${ }^{6}$, Nobuyuki Kanda ${ }^{7}$, Takahiro Tanaka ${ }^{8}$, \\ Kunihito Ioka' ${ }^{9}$, Kazuhiro Agatsuma ${ }^{4}$, Koh-suke Aoyanagi ${ }^{10}$, \\ Koji Arai $^{11}$, Akito Araya ${ }^{12}$, Hideki Asada ${ }^{13}$, Yoichi Aso ${ }^{4}$, \\ Takeshi Chiba $^{14}$, Toshikazu Ebisuzaki ${ }^{15}$, Yumiko Ejiri ${ }^{16}$, \\ Motohiro Enoki $^{17}$, Yoshiharu Eriguchi ${ }^{18}$, Masa-Katsu Fujimoto ${ }^{2}$, \\ Ryuichi Fujita $^{19}$, Mitsuhiro Fukushima ${ }^{20}$, Toshifumi Futamase ${ }^{21}$, \\ Tomohiro Harada $^{22}$, Tatsuaki Hashimoto ${ }^{5}$, Kazuhiro Hayama ${ }^{2}$, \\ Wataru Hikida $^{23}$, Yoshiaki Himemoto ${ }^{24}$, Hisashi Hirabayashi ${ }^{25}$, \\ Takashi Hiramatsu $^{26}$, Feng-Lei Hong ${ }^{27}$, Hideyuki Horisawa ${ }^{28}$, \\ Mizuhiko Hosokawa $^{29}$, Kiyotomo Ichiki $^{30}$, Takeshi Ikegami ${ }^{27}$, \\ Kaiki T Inoue $^{31}$, Koji Ishidoshiro ${ }^{4}$, Hideki Ishihara ${ }^{7}$,
}

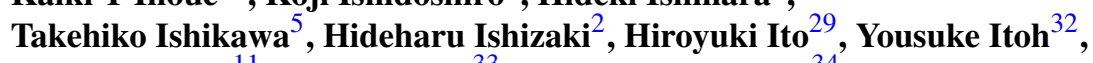
Kiwamu Izumi $^{11}$, Isao Kawano ${ }^{33}$, Nobuki Kawashima ${ }^{34}$, Fumiko Kawazoe $^{35}$, Naoko Kishimoto ${ }^{36}$, Kenta Kiuchi ${ }^{10}$, Shiho Kobayashi ${ }^{37}$, Kazunori Kohri ${ }^{38}$, Hiroyuki Koizumi ${ }^{5}$, Yasufumi Kojima $^{39}$, Keiko Kokeyama ${ }^{40}$, Wataru Kokuyama ${ }^{4}$, Kei Kotake ${ }^{41}$, Yoshihide Kozai ${ }^{42}$, Hiroo Kunimori ${ }^{43}$, Hitoshi Kuninaka ${ }^{5}$, Kazuaki Kuroda $^{26}$, Kei-ichi Maeda ${ }^{10}$, Hideo Matsuhara ${ }^{5}$, Yasushi Mino $^{44}$, Osamu Miyakawa ${ }^{26}$, Umpei Miyamoto ${ }^{45}$, Shinji Miyoki $^{26}$, Mutsuko Y Morimoto ${ }^{5}$, Toshiyuki Morisawa ${ }^{8}$, Shigenori Moriwaki ${ }^{46}$, Shinji Mukohyama ${ }^{47}$, Mitsuru Musha ${ }^{48}$, Shigeo Nagano $^{29}$, Isao Naito ${ }^{49}$, Kouji Nakamura ${ }^{2}$, Masahiro Nakamura ${ }^{48}$, Hiroyuki Nakano ${ }^{50}$, Kenichi Nakao ${ }^{7}$, Shinichi Nakasuka $^{51}$, Yoshinori Nakayama ${ }^{52}$, Kazuhiro Nakazawa ${ }^{4}$, Erina Nishida $^{16}$, Kazutaka Nishiyama ${ }^{5}$, Atsushi Nishizawa ${ }^{41}$, Yoshito Niwa $^{53}$, Taiga Noumi ${ }^{51}$, Yoshiyuki Obuchi ${ }^{20}$, Masatake Ohashi $^{26}$, Naoko Ohishi ${ }^{2}$, Masashi Ohkawa ${ }^{54}$, Kenshi Okada ${ }^{4}$, Norio Okada ${ }^{20}$, Kenichi Oohara ${ }^{55}$, Norichika Sago ${ }^{8}$, Motoyuki Saijo ${ }^{22}$, Ryo Saito $^{4}$, Masaaki Sakagami ${ }^{56}$, Shin-ichiro Sakai ${ }^{5}$, Shihori Sakata ${ }^{57}$, Misao Sasaki ${ }^{8}$, Takashi Sato ${ }^{58}$, Masaru Shibata ${ }^{8}$, Hisaaki Shinkai ${ }^{59}$, Kentaro Somiya $^{44}$, Hajime Sotani ${ }^{60}$, Naoshi Sugiyama ${ }^{30}$, Yudai Suwa ${ }^{4}$, Rieko Suzuki ${ }^{16}$, Hideyuki Tagoshi ${ }^{23}$, Fuminobu Takahashi ${ }^{47}$, Kakeru Takahashi ${ }^{4}$, Keitaro Takahashi $^{30}$, Ryutaro Takahashi $^{2}$, Ryuichi Takahashi $^{61}$, Tadayuki Takahashi $^{5}$, Hirotaka Takahashi $^{62}$, Takamori Akiteru $^{12}$, Tadashi Takano ${ }^{63}$, Keisuke Taniguchi ${ }^{32}$, Atsushi Taruya $^{64}$, Hiroyuki Tashiro $^{1}$, Yasuo Torii ${ }^{2}$, Morio Toyoshima ${ }^{29}$, Shinji Tsujikawa ${ }^{65}$, Yoshiki Tsunesada ${ }^{66}$, Akitoshi Ueda ${ }^{2}$, Ken-ichi Ueda $^{48}$, Masayoshi Utashima ${ }^{33}$, Yaka Wakabayashi ${ }^{16}$, 
Kent Yagi $^{1}$, Hiroshi Yamakawa ${ }^{67}$, Kazuhiro Yamamoto ${ }^{35}$, Toshitaka Yamazaki $^{68}$, Jun'ichi Yokoyama ${ }^{64}$, Chul-Moon Yoo ${ }^{69}$, Shijun Yoshida $^{21}$, Taizoh Yoshino ${ }^{70}$ and Ke-Xun Sun ${ }^{71}$

${ }^{1}$ Department of Physics, Kyoto University, Kyoto 606-8502, Japan

2 TAMA Project Office, National Astronomical Observatory of Japan, Mitaka, Tokyo 181-8588, Japan

${ }^{3}$ Faculty of Engineering, Hosei University, Koganei, Tokyo 184-8584, Japan

${ }^{4}$ Department of Physics, The University of Tokyo, Bunkyo, Tokyo 113-0033, Japan

5 Institute of Space and Astronautical Science, Japan Aerospace Exploration Agency, Sagamihara, Kanagawa 229-8510, Japan

${ }^{6}$ NASA Goddard Space Flight Center, Greenbelt, MD 20771, USA

${ }^{7}$ Department of Physics, Osaka City University, Osaka 558-8585, Japan

${ }^{8}$ Yukawa Institute for Theoretical Physics, Kyoto University, Kyoto 606-8502, Japan

${ }^{9}$ Institute of Particle and Nuclear Studies, KEK (High Energy Accelerator Research Organization), Tsukuba, Ibaraki 305-0801, Japan

${ }^{10}$ Department of Physics, Waseda University, Shinjuku, Tokyo 169-8555, Japan

${ }^{11}$ LIGO Project, California Institute of Technology, Pasadena, CA 91125, USA

12 Earthquake Research Institute, The University of Tokyo, Bunkyo, Tokyo 113-0032, Japan

${ }^{13}$ Department of Earth and Environmental Sciences, Hirosaki University, Hirosaki, Aomori 036-8560, Japan

${ }_{14}^{14}$ Department of Physics, Nihon University Setagaya, Tokyo 156-8550, Japan

15 RIKEN, Wako, Saitama 351-0198, Japan

16 Graduate School of Humanities and Sciences, Ochanomizu University, Bunkyo, Tokyo

112-8610, Japan

${ }^{17}$ Faculty of Business Administration, Tokyo Keizai University, Kokubunji, Tokyo 185-8502, Japan

${ }^{18}$ Graduate School of Arts and Science, The University of Tokyo, Meguro, Tokyo 153-8902, Japan

19 Theoretical Physics, Raman Research Institute, Sadashivanagar, Bangalore 560 080, India

${ }^{20}$ Advanced Technology Center, National Astronomical Observatory of Japan, Mitaka, Tokyo 181-8588, Japan

${ }^{21}$ Astronomical Institute, Tohoku University, Sendai, Miyagi 980-8578, Japan

${ }^{22}$ Department of Physics, Rikkyo University, Toshima, Tokyo 171-8501, Japan

23 Department of Earth and Space Science, Osaka University, Toyonaka, Osaka 560-0043, Japan

${ }^{24}$ College of Industrial Technology, Nihon University, Narashino, Chiba 275-8576, Japan

25 Wakabadai, Shiroyamacho, Sagamihara, Kanagawa 220-0112, Japan

${ }^{26}$ Institute for Cosmic Ray Research, The University of Tokyo, Kashiwa, Chiba 277-8582, Japan

${ }^{27}$ National Institute of Advanced Industrial Science and Technology (AIST), Tsukuba,

Ibaraki 305-8563, Japan

28 Department of Aeronautics and Astronautics, Tokai University, Hiratsuka, Kanagawa,

259-1292, Japan

${ }^{29}$ National Institute of Information and Communications Technology (NICT), Koganei, Tokyo 184-8795, Japan

${ }^{30}$ Department of Physics, Nagoya University, Nagoya, Aichi 464-8602, Japan

${ }^{31}$ School of Science and Engineering, Kinki University, Higashi-Osaka, Osaka 577-8502, Japan

32 Department of Physics, University of Wisconsin-Milwaukee, Milwaukee, WI 53201-0413, USA

33 Japan Aerospace Exploration Agency, Tsukuba, Ibaraki 305-8505, Japan

${ }^{34}$ Liaison Center, Kinki University, Higashi-Osaka, Osaka 577-8502, Japan

35 Max-Planck-Institute for Gravitational Physics (Albert-Einstein-Institute), Hannover, Germany

${ }^{36}$ Department of Aeronautics and Astronautics, Kyoto University, Uji, Kyoto 611-0011, Japan

37 Astrophysics Research Institute, Liverpool John Moores University, Egerton Wharf,

Birkenhead L41 1LD, UK

${ }^{38}$ Department of Physics, Tohoku University, Sendai, Miyagi 980-8578, Japan

39 Department of Physics, Hiroshima University, Higashi-hiroshima, Hiroshima 739-8526, Japan

${ }^{40}$ University of Birmingham, Edgbaston, Birmingham, B15 2TT, UK

${ }^{41}$ Division of Theoretical Astronomy, National Astronomical Observatory of Japan, Mitaka,

Tokyo 181-8588, Japan

42 Gunma Astronomical Observatory, Agatsuma, Gunma 377-0702, Japan 
${ }^{43}$ National Institute of Information and Communications Technology (NICT), Bunkyo, Tokyo, 113-0001 Japan

44 Theoretical Astrophysics, California Institute of Technology, Pasadena, CA 91125, USA

${ }^{45}$ Institute of Theoretical Physics, Rikkyo University, Toshima, Tokyo 171-8501, Japan

${ }^{46}$ Department of Advanced Materials Science, The University of Tokyo, Kashiwa,

Chiba 277-8561, Japan

47 Institute for Physics and Mathematics of the Universe (IPMU), The University of Tokyo,

Kashiwa, Chiba 277-8568, Japan

${ }^{48}$ Institute for Laser Science, The University of Electro-Communications, Chofu, Tokyo 182-8585, Japan

${ }^{49}$ Numakage, Saitama, Saitama 336-0027, Japan

${ }^{50}$ Rochester Institute of Technology, Rochester, NY 14623, USA

${ }^{51}$ Department of Engineering, The University of Tokyo, Bunkyo, Tokyo 113-8656, Japan

52 Department of Aerospace Engineering, National Defense Academy, Yokosuka,

Kanagawa 239-8686, Japan

53 JASMINE Project Office, National Astronomical Observatory of Japan, Mitaka, Tokyo 181-8588, Japan

${ }^{54}$ Department of Biocybernetics, Niigata University, Niigata 950-2181, Japan

${ }^{55}$ Department of Physics, Niigata University, Niigata 950-2181, Japan

${ }^{56}$ Graduate School of Human and Environmental Studies, Kyoto University, Kyoto 606-8501, Japan

${ }_{57}$ Observatoire de la Cote d'Azur, Le Mont Gros, Nice Cedex 4, France

${ }^{58}$ Department of Electrical and Electronic Engineering, Niigata University, Niigata 950-2181, Japan

${ }^{59}$ Department of Information Systems, Osaka Institute of Technology, Hirakata, Osaka

573-0196, Japan

${ }^{60}$ Institute for Astronomy and Astrophysics, Eberhard Karls University of Tuebingen,

72076 Tuebingen, Germany

${ }^{61}$ Faculty of Science and Technology, Hirosaki University, Hirosaki, Aomori 036-8561, Japan

${ }^{62}$ Department of Management and Information Systems Science, Nagaoka University of

Technology, Nagaoka, Niigata 940-2188, Japan

${ }^{63}$ Department of Electronics and Computer Science, Nihon University, Funabashi,

Chiba 274-8501, Japan

${ }^{64}$ Research Center for the Early Universe, The University of Tokyo, Bunkyo, Tokyo 113-0033, Japan

${ }^{65}$ Department of Physics, Tokyo University of Science, Shinjuku, Tokyo, 162-8601, Japan

${ }^{66}$ Graduate School of Science and Engineering/Physics, Tokyo Institute of Technology, Meguro,

Tokyo 152-8550, Japan

${ }^{67}$ Research Institute for Sustainable Humanosphere, Kyoto University, Uji, Kyoto 611-0011, Japan

${ }^{68}$ ALMA Project Office, National Astronomical Observatory of Japan, Mitaka, Tokyo 181-8588, Japan

${ }^{69}$ Asia Pacific Center for Theoretical Physics, Pohang, Gyeongbuk 790-784, Korea

${ }^{70}$ Nakamura-minami, Nerima, Tokyo 176-0025, Japan

${ }^{71}$ Stanford University, Stanford, CA 94305-4088, USA

E-mail: ando@ scphys.kyoto-u.ac.jp

Received 31 December 2009, in final form 1 March 2010

Published 6 April 2010

Online at stacks.iop.org/CQG/27/084010

\section{Abstract}

A space gravitational-wave antenna, DECIGO (DECI-hertz interferometer Gravitational wave Observatory), will provide fruitful insights into the universe, particularly on the formation mechanism of supermassive black holes, dark energy and the inflation of the universe. In the current pre-conceptual design, DECIGO will be comprising four interferometer units; each interferometer unit will be formed by three drag-free spacecraft with $1000 \mathrm{~km}$ separation. 
Since DECIGO will be an extremely challenging mission with high-precision formation flight with long baseline, it is important to increase the technical feasibility before its planned launch in 2027. Thus, we are planning to launch two milestone missions. DECIGO pathfinder (DPF) is the first milestone mission, and key components for DPF are being tested on ground and in orbit. In this paper, we review the conceptual design and current status of DECIGO and DPF.

PACS numbers: $04.30 . \mathrm{Tv}, 04.80 . \mathrm{Nn}$, 95.55.Ym, 95.85.Sz

(Some figures in this article are in colour only in the electronic version)

\section{Introduction}

Observation of the universe with gravitational waves (GWs) will bring us new astronomical and cosmological knowledge which are completely different ones from, or complementary ones with, those obtained by traditional observation with electro-magnetic waves $[1,2]$. In order to open this new window to the Universe, several GW detectors are under operation, and observation data are being accumulated with sufficient sensitivities to detect GWs from nearby galaxies [3-6]. However, the detection rate with these detectors is estimated to be small, much lower than one event-per-year. Thus, several future ground-based and space detectors have been proposed so as to detect GWs with higher rate, and to establish new field of GW astronomy.

There are two paths to observe GWs with higher rate in future detectors. One path is to improve the sensitivity of ground-based detectors. Ground-based detectors mainly target at high-frequency $(10 \mathrm{~Hz}-1 \mathrm{kHz}) \mathrm{GWs}$ radiated from violent astronomical events, such as inspiral-and-mergers of neutron-star binaries, and supernova explosions with gravitational core collapses. The expected number of GW detections will be increased by expanding the observable range with improved sensitivity. In next-generation detectors, which will start operation in this decade, such as Advanced LIGO [7], Advanced VIRGO [8] and LCGT [9], more than a few detections are expected with 1 year observation. Another path to detect GWs with higher rate is to observe GWs in lower frequency bands. In these bands, we can expect a larger amplitude or stationary GWs from mergers of massive black holes, from the early Universe, and so on. Space detectors are superior in low-frequency observations below $1 \mathrm{~Hz}$, which is difficult to access by terrestrial detectors because of the gravity disturbances of the earth. LISA (Laser Interferometer Space Antenna) [10] has an observation band around $1 \mathrm{mHz}$. DECIGO (DECi-hertz Interferometer Gravitational wave Observatory) [11, 12] and BBO (Big Bang Observer) [13], which will be following missions of LISA, are space interferometric detectors with observation bands around $0.1 \mathrm{~Hz}$. Since this band is between that of LISA and terrestrial detectors, different or complementary information on the GW sources will be obtained by DECIGO.

Since DECIGO will be an extremely large mission both in its scale and required resources, it is important to increase the technical feasibility step-by-step. We have a roadmap to launch two milestone missions. DECIGO pathfinder (DPF) [14] is the first milestone mission to test the key technologies with a single spacecraft in a low earth orbit, and to carry out observation runs. In this paper, we review the scientific objectives, the conceptual design and current status of DECIGO and DPF. 


\section{DECIGO}

DECIGO (DECI-hertz interferometer Gravitational wave Observatory) is a future space gravitational-wave (GW) antenna, with an observation frequency band of around $0.1 \mathrm{~Hz}$. This band opens the possibility of observing GWs from the cosmological distance, because it is free from the confusion noises by irresolvable GW signals from too many white dwarf binaries [15]. With an extremely good sensitivity, DECIGO would enable us to detect GWs from the very early Universe, which could provide important information to understand the beginning of the Universe.

\subsection{Scientific targets}

The purpose of DECIGO is to obtain new knowledge on the history of the Universe and the formation mechanism of galaxies by GW observation. For this purpose, DECIGO has three main target GW sources: intermediate-mass black hole mergers, distant neutron-star binaries and stochastic background GWs from the early Universe.

Since frequencies of radiated GW signals are determined by the revolution time scales or masses of the sources, the mass range of the DECIGO's targets is between those of groundbased detectors and LISA. DECIGO targets at GW sources from intermediate mass scale phenomena, in particular from binary inspiral and mergers of intermediate mass $\left(10^{3}-10^{5} M_{\odot}\right)$ black holes. According to some theories, a supermassive black hole which inhabits at the center of galaxy has been formed from multiple collisions of intermediate-mass black holes [16]. With DECIGO sensitivity, a merger event of $10^{3} M_{\odot}$ black holes at $z \sim 1$ will be observed with a signal-to-noise ratio of 6000. Observation by DECIGO could reveal the mechanism of the formation of super-massive black holes at the center of galaxies.

DECIGO also targets at GWs from neutron-star binaries; GW signals will be in the DECIGO band a few months to 5 years before merger. Thus, DECIGO can be a predictor for these merger events, which would be helpful for observations by ground-based detectors [17]. In addition, DECIGO will have sufficient sensitivity to observe GWs from distant (redshift 1) neutron-star binaries. By resolving GW signals emitted from many (about $3 \times 10^{5}$ ) binaries in this range, we will obtain information of mass distribution of neutron stars, and thus, on the theory of the evolution of massive stars and on the equation of state of high-density matters [18]. Moreover, observing binaries at cosmological distance, which play as precise clocks, it will be possible to determine cosmological parameters with high precision $[11,19]$.

The most exciting target of DECIGO will be the stochastic background GWs from the early Universe; GWs could be originated from inflation [20], primordial black holes [21], astrophysical objects [22] and so on. It will be hard to observe these targets by electromagnetic waves directly because of scattering in high-energy plasma in the early Universe. So as to distinguish the stochastic background GWs from detector noises, DECIGO has multiple interferometer units for cross-correlation observation.

\subsection{Conceptual design}

In the current pre-conceptual design, a unit of DECIGO is formed by three drag-free spacecraft, $1000 \mathrm{~km}$ apart from one another (figure 1, left). These spacecraft have test mass mirrors inside them, which form long-baseline Fabry-Perot (FP) cavities; gravitational waves will be observed as changes in the cavity lengths. We adopted an FP interferometer configuration because it provides a better sensitivity at $0.1 \mathrm{~Hz}$ band than an optical transponder configuration used in LISA [10]. The distance between spacecraft (FP cavity arm length) was chosen to 


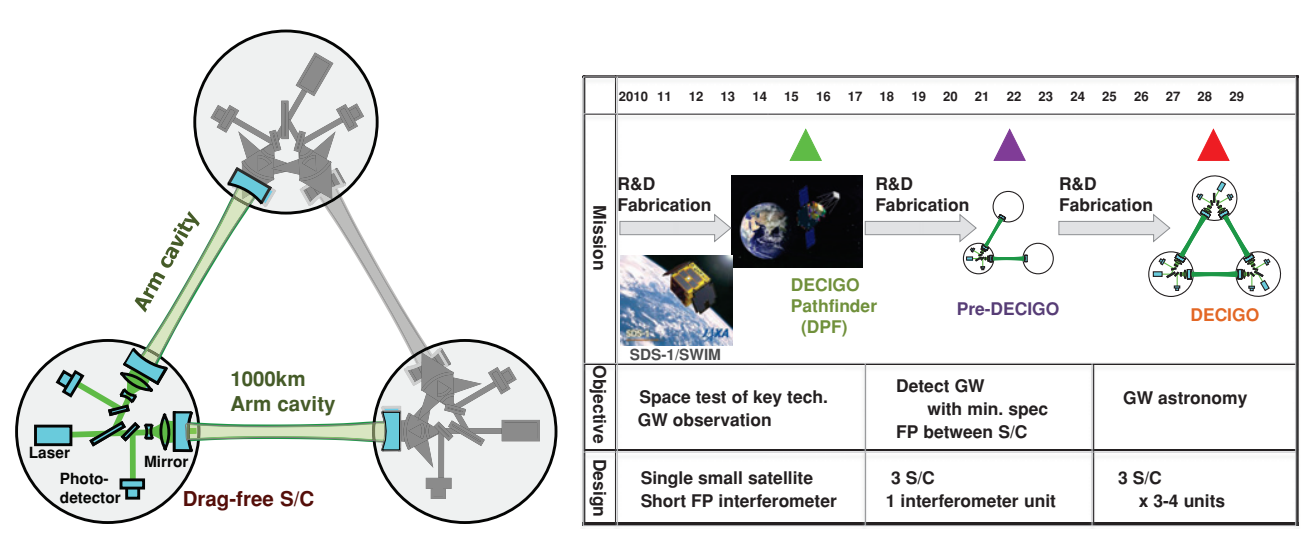

Figure 1. Conceptual design of DECIGO (left) and roadmap to realize DECIGO (right).

be $1000 \mathrm{~km}$ so as to be short enough to avoid diffraction losses of laser power, and to store sufficient laser power inside the cavities, and yet to be long enough to ensure the high sensitivity for GW signals. The mirrors forming the cavities have a diameter of $1 \mathrm{~m}$ with moderate reflectivity to realize the cavity finesse of 10 . The mass of mirror (about $100 \mathrm{~kg}$ ) was chosen to be the largest we would fabricate and handle. The laser source of DECIGO will have an effective power of $10 \mathrm{~W}$ with a wavelength of $532 \mathrm{~nm}$. The orbit and constellation of DECIGO are to be determined, considering the gravity disturbances by the sun and planets, durability of the thruster fuels, solar power supply and the required angle resolution for the GW source, and so on. One of the candidates of the orbit is a record-disk orbit around the sun, along the earth orbit.

\subsection{Roadmap}

DECIGO will be an extremely large mission both in its scale and required resources; four interferometer units will orbit around the sun along the earth orbit, and each unit will be formed by three drag-free spacecraft that are separated by $1000 \mathrm{~km}$ from one another. Thus, a long and intensive development phase will be required in order to realize DECIGO (figure 1, right). We plan to launch DECIGO in 2027 after design, research and development with ground-based experiments. We also have two milestone space missions, DECIGO pathfinder (DPF) and Pre-DECIGO, before DECIGO. The purpose of Pre-DECIGO [23] is to detect GWs with minimum specifications. Pre-DECIGO will have a down-sized configuration of DECIGO, comprising three spacecraft with shorter separation of $100 \mathrm{~km}$. The purpose and design of DPF are described in the next Section.

\section{DECIGO pathfinder}

DECIGO pathfinder (DPF) will be a small satellite orbiting the earth. The mission payload part of DPF is designed to be a prototype of DECIGO and to test the key technologies of DECIGO, comprising a short Fabry-Perot (FP) cavity, a stabilized laser source and a drag-free control system. In addition, DPF has its own scientific goals: observation of gravitational waves and the gravity of the earth, and demonstrations of advanced space technologies. 


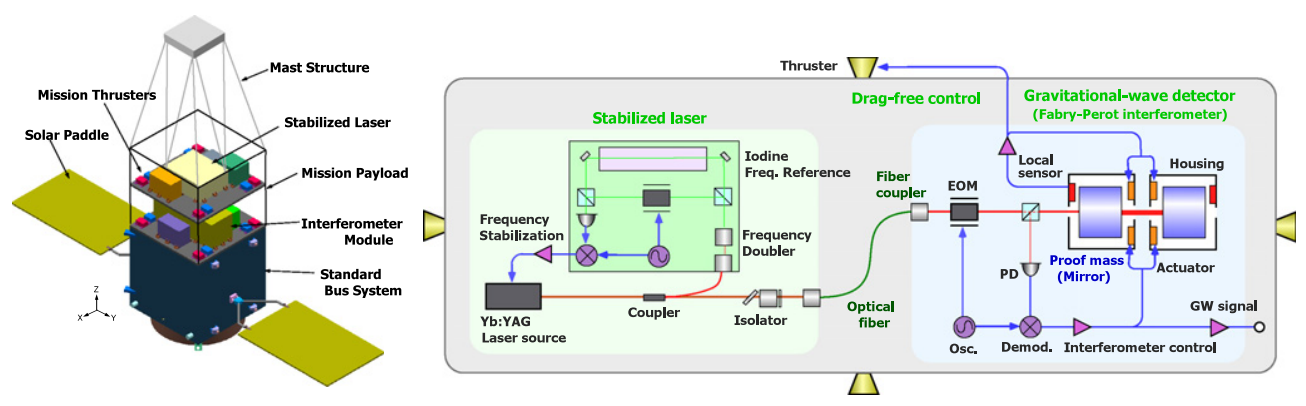

Figure 2. Overview of the DPF satellite (left) and the diagram of the mission payload of DPF (right).

\subsection{DPF satellite and bus system}

Conceptual design of DPF is shown in figure 2 (left); DPF will be a single satellite with weight of about $350 \mathrm{~kg}$, orbiting the earth with an altitude of $500 \mathrm{~km}$. DPF will be launched by a next-generation solid propellant rocket, which is being developed as a successor of the M-V launch vehicle of JAXA (Japan Aerospace Exploration Agency). For stable power generation and temperature equilibrium of the satellite, DPF will have a circular sun-synchronous dawnto-dusk orbit. Two proof masses inside the satellite will trail along the same orbit. The earth-synchronous attitude of the satellite will be passively stabilized by the gravity gradient of earth, and actively controlled by a drag-free control system to cancel external disturbances. For this control, small mission thrusters will be used; momentum wheels will not be loaded so as to avoid their mechanical disturbances.

The DPF satellite will be formed by the combination of a bus module and a mission payload module. We are planning to use a standard bus module under development in JAXA, which has a weight of about $200 \mathrm{~kg}$, and a size of $950 \times 950 \times 1100 \mathrm{~mm}$. This bus will provide a $940 \mathrm{~W}$ power with four solar-cell puddles, and $2 \mathrm{Mbps}$ downlink rate. A mission module will be attached to the upside of the bus module. The data processing system is based on a SpaceWire-based communication standard. The bus and mission modules are to be connected with power lines and SpaceWire communication lines, and cables for temperature controls.

\subsection{DPF mission design}

The mission payload part of DPF will have a size of $950 \times 950 \times 900 \mathrm{~mm}$, and a mast structure for gravity-gradient attitude stabilization will be attached at the top of the module. The mission payload of DPF is designed to be a prototype of DECIGO, comprising a short FP cavity, a stabilized laser source and a drag-free control system (figure 2, right).

The FP cavity is formed by two mirrors which act as free proof masses. Each mirror is to be placed inside a module called housing. The housing will have electrostatic-type local sensors and actuators on its frame, which are to be used to monitor and to control the relative motion between the mirror and the frame in all degrees of freedom. In addition, the housing will have a function of a launch lock, which will clump the mirror at the launch of the satellite and release it in the orbit with a small initial velocity. The cavity has a baseline length of about $30 \mathrm{~cm}$ and a finesse of about 100. The length change in the FP cavity, which would be caused by gravitational waves or external disturbances, is measured by means of a stabilized laser beam. 
As a light source, we will use an Yb:YAG laser in which the frequency is stabilized using the iodine absorption line. The requirement for the frequency stabilization is $0.5 \mathrm{~Hz} \mathrm{~Hz}-1 / 2$. The laser source has an output power of $100 \mathrm{~mW}$ at a wavelength of $1030 \mathrm{~nm}$. The drag-free control of the satellite will work as a shield against external forces caused by solar radiation and dragged by residual atmosphere. Drag-free control will be realized by measuring the relative fluctuations between the mirrors and the satellite, and basically feeding these signals back to the satellite position using low-noise mission thrusters. The satellite motion will be controlled in all degrees of freedom with a bandwidth of about $10 \mathrm{~Hz}$. DPF has two test mass mirrors inside it; roughly, the common motion signals of two test masses will be used for the drag-free control of the satellite, and the differential motion signals will be fed back to the test masses so as to stabilize the FP cavity.

\subsection{Objectives of DPF}

DPF has its own scientific objectives as well as technical tests for DECIGO. One is observation of gravitational waves and gravity of the earth, and another is space demonstration of advanced precision-measurement techniques.

3.3.1. Scientific observation. Two proof masses of DPF will be kept inside the spacecraft untouched to avoid external disturbances, and to be sensitive to gravitational forces, such as gravitational waves and earth's gravity distributions. Gravitational waves would be detected as tidal-force fluctuations on two proof masses, which are measured by a laser interferometer. The DPF interferometer will have a sensitivity of $2 \times 10^{-15} \mathrm{~Hz}^{-1 / 2}$ at around $1 \mathrm{~Hz}$ frequency band, which corresponds to a GW sensitivity limit of about $h \sim 10^{-15}$. At this frequency band, it is expected that gravitational waves are radiated from intermediate-mass-black hole inspirals and mergers. The observable ranges of DPF for these phenomena are estimated to cover our galaxy. DPF has a potential to detect gravitational-wave signals, if there is an inspiral-and-merger event with $10^{3}-4 \times 10^{5} M_{\odot}$ black holes in our galaxy. Although the probability of having such events is considered to be rare, data obtained by DPF observations will have importance because this observation band is difficult to access by ground-based gravitational-wave detectors and other space-based detection methods. The analysis of DPF data may be more complicated than that for ground-based detectors because of satellite orbital motion and the effect of the earth. This will be a good challenge for future space detectors.

Observation of the gravity of the earth is another scientific objective of DPF. Since the proof masses orbit the earth almost freely, gravity distributions of the earth would be observed from the trajectories of the proof masses. In order to cancel the drag force by air and solar radiation, the relative displacements between the proof masses and the satellite frame are to be measured by small Michelson-interferometer-type laser sensors with an acceleration sensitivity of $10^{-11} \mathrm{~m} \mathrm{~s}^{-2}$. Several satellites to observe the earth's gravity have been launched so far [24]. However, there is a concern for an observation gap time between the ends of current missions and the launch of next-generation missions. DPF will be able to bridge the gap time with comparable sensitivity.

3.3.2. Advanced technologies of precise measurement in space. DPF will have a precision position measurement system with an FP interferometer, a highly stabilized laser source and a suppression system of satellite fluctuation with drag-free control. These are advanced space technologies with scientific significance. In addition, the combination of these technologies will be useful for other future precision measurement missions in a zero-gravity environment. 
The main FP interferometer in DPF with a baseline length of $30 \mathrm{~cm}$ is designed as a prototype of $1000 \mathrm{~km}$ arm cavity of DECIGO. Although measurement and operation with such an interferometer are a well-established technique in a ground-based environment, there is no example of an FP cavity formed by free floating mirrors in a space environment. In an FP configuration, we can expect better sensitivity than that of a Mach-Zender interferometer, which is used in LISA pathfinder (LPF) [25]. The sensitive operation of the FP interferometer (with a displacement sensitivity of $6 \times 10^{-16} \mathrm{~m} \mathrm{~Hz}^{-1 / 2}$ ) in DPF will provide a new possibility for precise measurements in a space environment.

In DPF, the frequency noise of the laser source is stabilized using saturated absorption spectroscopy of iodine molecules, targeting at a stability of $0.5 \mathrm{~Hz} \mathrm{~Hz}^{-1 / 2}$ in the $0.1 \mathrm{~Hz}$ frequency band. Frequency stabilization of a laser source has been well studied for groundbased gravitational-wave detectors, and in other fields, such as precise metrology, spectroscopy, optical communications and so on. On the other hand, there are few experiments in a space environment. However, recently, laser sources with high-frequency stability have come to be required in space missions. LISA requires $30 \mathrm{~Hz} \mathrm{~Hz}^{-1 / 2}$ frequency stability. DPF will show a new potential of a high-stabilized laser in space.

The fluctuation of the DPF satellite will be stabilized smaller than $10^{-9} \mathrm{~m} \mathrm{~Hz}^{-1 / 2}$ by drag-free control. Drag-free control of a satellite was realized by the TRIAD-I satellite in 1972 for the first time, and by several follow-on satellites for investigating a navigation system. Recently, drag-free control was also realized by the Gravity Probe-B satellite for tests of the general theory of relativity [26]. LPF will demonstrate drag-free control at the Lagrange 1 (L1) point between the earth and the sun, at which the gravitational environment is stable. DPF will demonstrate it in an earth orbit, with the help of passive attitude stabilization by gravity gradient of the earth. This will be an original scheme, which may open a new possibility in the application of a zero-gravity environment in space.

\subsection{Current status of DPF}

Currently, DPF is one of the candidates of small satellite missions of JAXA. JAXA has a project to launch at least three small satellites in the next 5 years from 2012, using standard bus systems. The first mission has been selected to be SPRINT-A/EXCEED, which is an UV telescope for planet observation. EXCEED will be launched in 2012. The second mission has decided to be ERG (energization and radiation in geospace). DPF is one of mission candidates for the third mission, with the target launch in 2015.

Research and development are underway with the support of JAXA, mainly concerning a mission studies including satellite design and drag-free control topology, and tests of key devices, such as a housing system for a proof mass, a stabilized laser and thrusters. In addition, a small demonstration module, named SWIM (SpaceWire Interface demonstration Module), has been developed and launched on 23 January 2009 in SDS-1, a JAXA's technology demonstration satellite. SWIM contains a space-qualified data processor and recorder with the SpaceWire interface, and a tiny gravitational-wave detector module with a size of $160 \times 80 \times$ $80 \mathrm{~mm}$. SWIM has been operated in orbit successfully for a year, providing heritages for DPF on a SpaceWire-based data processing system and on sensing and control of proof masses in a space environment.

\section{Conclusion}

DECIGO will establish a new window for gravitational-wave astronomy, and the DECIGO pathfinder (DPF) will be the first significant milestone mission to test the key technologies 
for DECIGO. Moreover, DPF will provide new scientific knowledge on gravitational-wave observations, on the gravity of the earth and on advanced technologies of precise measurements in a space environment. DPF has been selected as one of the candidates of small satellite missions of JAXA, and research and development for key components are underway. We are hoping to launch DPF in 2015 in the best case.

\section{Acknowledgments}

This research was supported by the Japan Aerospace Exploration Agency (JAXA), by the Japan Society for the Promotion of Science (JSPS), grant-in-aid for scientific research, by the Global COE Program of the graduate school of science in Kyoto University and by the Research Center for the Early Universe (RESCEU) in the University of Tokyo.

\section{References}

[1] Thorne K S 1987 Gravitational radiation Three Hundred Years of Gravitation ed S Hawking and W Israel (Cambridge: Cambridge University Press) pp 330-458

[2] Sathyaprakash B S and Schutz B F 2009 Living Rev. Relativ. 122

[3] Abramovici A et al 1992 Science 256325

[4] The VIRGO Collaboration 1997 VIRGO Final Design Report

[5] Danzmann K et al 1994 Proposal for a 600 m Laser-interferometric gravitational wave antenna Max-Planck Institut fuer Quantenoptik Report 190 Garching, Germany

[6] Tsubono K 1995 300-m laser interferometer gravitational wave detector (TAMA300) in Japan Gravitational Wave Experiments ed E Coccia, G Pizzella and F Ronga (Singapore: World Scientific) p 112-4 Ando M et al 2001 Phys. Rev. Lett. 863950

[7] LIGO Scientific Collaboration P Fritschel (ed) LIGO Technical Note T010075 Weinstein A 2002 Class. Quantum Grav. 191575

[8] Acernese F et al 2008 J. Opt. A 10064009

[9] Kuroda K et al 2006 Class. Quantum Grav. 23 S19

[10] LISA 2000 Laser interferometer space antenna: a cornerstone mission for the observation of gravitational waves System and Technology Study Report, ESA-SCI p 11

[11] Seto N, Kawamura S and Nakamura T 2001 Phys. Rev. Lett. 87221103

[12] Kawamura S et al 2006 Class. Quantum Grav. 23 S125

[13] Phinney S et al 2004 The big bang observer: direct detection of gravitational waves from the birth of the Universe to the present NASA Mission Concept Study

Crowder J and Cronish N J 2005 Phys. Rev. D 72083005

[14] Ando M et al 2009 Class. Quantum Grav. 26094019

[15] Evans C R, Iben I and Smarr L 1987 Astrophys. J. 323129

Liu J 2009 Mon. Not. R. Astron. Sci. 4001850 and references therein

[16] Sesana A, Gair J, Mandel I and Vecchio A 2009 Astrophys. J. 698 L129-32

Matsubayashi T, Makino J and Ebisuzaki T 2007 Astrophys. J. 656 879-96

[17] Takahashi R and Nakamura T 2003 Astrophys. J. 596 L231-4

[18] Cutler C and Harms J 2006 Phys. Rev. D 79042001

[19] Cutler C and Holz D 2009 Phys. Rev. D 80104009

[20] Smith T L, Kamionkowski M and Cooray A 2006 Phys. Rev. D 73023504 Kudoh H et al 2006 Phys. Rev. D 73064006 Kuroyanagi S, Chiba T and Sugiyama N 2009 Phys. Rev. D 79103501

[21] Saito R and Yokoyama J 2009 Phys. Rev. Lett. 102161101

[22] Suwa Y et al 2007 Astrophys. J. 665 L43-6

[23] Sato S et al 2008 J. Phys. 154012040

[24] GRACE mission web page http://www.csr.utexas.edu/grace Champ mission web page http://op.gfz-potsdam.de/champ/index_CHAMP.html GOCE mission web page http://www.esa.int/esaLP/LPgoce.html

[25] Armano M et al 2009 Class. Quantum Grav. 26094001

[26] Buchman S et al 1998 Proc. of the 32nd COSPAR Scientific Assembly in Nagoya, Japan Buchman S et al 2000 Adv. Space Res. 251177 